\title{
Old data and friends improve with age: Advancements with the updated tools of
} GeneNetwork

\author{
Alisha Chunduri ${ }^{1}$, David G. Ashbrook ${ }^{2}$ \\ ${ }^{1}$ Department of Biotechnology, Chaitanya Bharathi Institute of Technology, Hyderabad 500075, \\ India \\ ${ }^{2}$ Department of Genetics, Genomics and Informatics, University of Tennessee Health Science \\ Center, Memphis, TN 38163, USA
}

\begin{abstract}
Understanding gene-by-environment interactions is important across biology, particularly behaviour. Families of isogenic strains are excellently placed, as the same genome can be tested in multiple environments. The BXD's recent expansion to 140 strains makes them the largest family of murine isogenic genomes, and therefore give great power to detect QTL. Indefinite reproducible genometypes can be leveraged; old data can be reanalysed with emerging tools to produce novel biological insights.
\end{abstract}

To highlight the importance of reanalyses, we obtained drug- and behavioural-phenotypes from Philip et al. 2010, and reanalysed their data with new genotypes from sequencing, and new models (GEMMA and R/qt12). We discover QTL on chromosomes 3, 5, 9, 11, and 14, not found in the original study.

We narrowed down the candidate genes based on their ability to alter gene expression and/or protein function, using cis-eQTL analysis, and variants predicted to be deleterious. Co-expression analysis ('gene friends') and human PheWAS were used to further narrow candidates.

Prominent candidate genes include: Slitrk6 in a Chr 14 QTL for locomotion in the center of an open field, we show to be part of a coexpression network involved in voluntary movement, and association with neuropsychiatric phenotypes in PheWAS; and Cdk14, one of only 3 genes in a Chr 5 QTL for handling induced convulsions after ethanol treatment, that is regulated by the anticonvulsant drug valproic acid.

By using families of isogenic strains, we can reuse and reanalyse data to discover novel and highly plausible candidate genes involved in response to the environment. 


\section{Introduction}

Two of the biggest problems in analyses of biomedical data are irretrievability and irreplicability. Biomedical data is often lost as soon as it is published, locked within a forgotten harddrive, or siloed in a little-used format on a lab's website. There are many efforts to make data publicly retrievable, such as the FAIR Principles [1], and these are allowing the combined analysis of many datasets, and reanalysis using new tools. There is still the problem of irreducible datasets: for example if a sample from a particular outbred cohort is found to be an outlier during data analysis, there is no way to go back to that genometype and remeasure the phenotype. Nor can new phenotypes be measured in the same individuals in the same environments at a later date as new tools emerge. Families of isogenic strains solve this problem, giving us reproducible genometypes that can be sampled many times, under many environmental conditions: so called experimental precision medicine [2]. This means that a genometype sampled 30 years ago in a different country, can be replicated now, in any lab, with any environmental variable of interest, using any technique. The GeneNetwork.org website allows this combination of FAIR data and reproducible genomes, meaning that we can now go back to previous datasets, and reanalyze them with new data and new tools. Every new dataset adds exponentially to the number of possible connections. In this paper, we will reanalyze drug and addiction related data from over a decade ago using new genotypes for the BXD family and new tools, showing that we can identify new quantitative trait loci (QTLs) and highly plausible candidate genes.

Quantitative trait locus (QTL) mapping has been carried out in numerous species to associate regions of the genome to phenotypes, even before the structure of the genome was well understood (e.g. [3]). Rodents, especially mice, have been the species most prominently used for biomedically relevant traits. Amongst these, the BXD family of recombinant inbred (RI) mouse strains, have been extensively used for almost 50 years in fields such as neuropharmacology $\quad[4,5,6,7,8,9,10]$, immunology $\quad[11,12,13,14,15]$, behavior $[16,17,18,19,20,21,22,23]$, aging $[24,25,26,27,28]$, neurodegeneration $[29,30,31,32,33]$, and gut microbiome-host interactions [34].

The development of the BXD panel was started by Benjamin A. Taylor by inbreeding the progeny of female $\mathrm{C} 57 \mathrm{BL} / 6 \mathrm{~J}$ and male $\mathrm{DBA} / 2 \mathrm{~J}$ strains, for the purpose of mapping Mendelian traits [35]. This led to the original 32 BXD strains, which now carry the suffix 'TyJ' (Taylor to Jackson Laboratory). To increase the power and precision of QTL mapping, the number of strains has been expanded [36], including through advanced intercross by [37], and now to a total of 140 extant strains [2], making this resource the largest family of murine isogenic strains. Phenotypes in the BXD have been measured under many conditions, allowing identification of gene-by-environment interactions. Understanding these interactions can potentially help in the discovery of complex therapeutic solutions, and are a vital part of the development of precision 
medicine.

GeneNetwork.org is a tool for quantitative genetics that started in 2001 as WebQTL [38]. It evolved from analyses of forward genetics in the BXD mouse family, to phenome-wide association studies and reverse genetics in a variety of species. Although GeneNetwork contains data for many species and populations, it most prominently contains data for the BXD family. Over 10,000 'classical' phenotypes, measured under a variety of environmental conditions, and over 100 'omics datasets are available on GeneNetwork for the BXD family. GeneNetwork and BXD RI population are therefore a powerful tool for systems genetics and experimental precision medicine.

The great advantage of inbred lines, with stable genome-types that can be resampled, is that data can be reused and reanalyzed over time, as tools improve. From the very start of the genome sequencing revolution, when loci were first mapped to causative genes, new tools and a greater understanding of the genome have allowed us to go back to old data and gain new insight.

In this study, we will demonstrate how new biological insight into drugs of abuse can be gained by reanalyzing data in the BXD family, using improved genotypes from sequencing, and new mapping methods (linear mixed models). Using this method, we have discovered new QTLs and candidate genes for behavioral phenotypes associated with the predisposition of drug- and behavior-related traits obtained from Philip et al. 2010 [39].

\section{Methods}

\section{Phenotype data}

The traits used for analysis in this study were acquired by Philip, and the team and published in 2010 [39]. All data from this publication are freely available on GeneNetwork.org, and were obtained from the BXD published phenotypes. Philip's study aimed to determine the influence of genes in the response to environment and plausibility of similar interaction with the drug-related attributes including response to and, withdrawal from cocaine, 3,4-methylenedioxymethamphetamine, morphine, and ethanol and their correlation to traits including anxiety, locomotion, stress sensitivity, and pain sensitivity. Complex phenotyping batteries consisting of diverse behavioral assays were employed on the RI strains and multi-variate analyses were performed using GeneNetwork.org. An interplay between environmental factors, drug-induced neural changes, and genetic factors underlie the predisposition of an individual to addiction. In our study, a total of 762 traits were analyzed [Supplementary table 1] using new genotypes and the GEMMA mapping software, to identify novel candidate genes and gene-by-treatment interactions. We did not include morphine related traits, as these are being actively studied by others. Of the then extant population of 79 
strains [7], Philip's study used approximately 70 strains to measure the traits.

\section{New genotypes from sequencing}

A total of 152 BXD strains have now been sequenced using linked-read technologies, and new genotypes for all 152 BXD strains have been produced from this (Personal communications). Variants were chosen to define the start and end of each haplotype block, and variant positions from the previously published genotypes were kept to allow maximum back compatibility with previous publications.

\section{GEMMA, kinship within the BXD and QTL mapping}

The BXD family has been produced in several 'epochs' across 40 years, using both standard F2 recombinant inbred methods, and advanced intercross recombinant inbred methods [2]. This has led to both expected and unexpected kinship between BXD strains. This kinship between strains can lead to bias, as it breaks the expectations of previously used methods, such as the Haley-Knott mapping algorithm that was used by Philip's study. Updated linear mixed models including Genome-wide Efficient Mixed Model Association (GEMMA) and R/qt/2 accessible in the GeneNetwork have been used for this study, as they allow correction for kinship, as well as other cofactors if needed.

Analysis of the 762 traits (taken from Philip et al. study) was carried out using the GEMMA mapping tool with the genotypes from sequencing, a minor allele frequency (MAF) of 0.05 and utilizing the Leave One Chromosome Out (LOCO) method. This computation provides a $-\log (p)$ value between each marker and the phenotype. We used a $-\log (p)>4$, as significant. However, since permutations of the GEMMA algorithm are not currently available in GeneNetwork, we confirmed the significance of these QTL using the linear mixed model tool within r/QTL2 [40], with 5000 permutations of the data.

\section{Identification of novel QTL}

Two methods were used to identify significant QTL.

Firstly, traits with an adjusted $p<0.05$ using permutation in r/QTL2 (described above), as these are significant after empirical correction.

The second method we used was to take advantage of independent traits which share QTL at the same location with suggestive $p$-values $(p<0.63)$. This $p<0.63$ equates to one false positive per genome scan. However, the likelihood of any particular chromosome having a QTL on it is approximately 1 in 20 (i.e. $p<0.05$ ) due to there being 20 chromosomes in mice. The likelihood of two independent traits sharing the same QTL location by chance is therefore much lower than $p<0.05$. Traits were called as independent if they were carried out in separate groups of 
animals (e.g. males and females) or if the traits were measured at independent timepoints (e.g. at 10 minutes after treatment and 60 minutes after treatment).

\section{QTL confidence intervals}

A 1.5 LOD or $1.5-\log (p)$ drop was used to determine the QTL confidence interval for each statistically significant trait (in our particular case of a two-parent population LOD and $-\log (p)$ are approximately equal). Therefore, for each of the QTL above [Supplementary table 2] we were able to generate a list of genes within this confidence interval. Genes were called within our QTL interval using the genenetwork QTL mapping tool - this provides both protein coding genes, non-coding genes, and predicted gene models.

\section{Cis-eQTL mapping}

A cis-eQTL indicate that a variant within or very close to a gene influences its expression. Genes with cis-eQTLs are high priority candidates, as it provides a potential causal pathway between the gene variant and the phenotype of interest (i.e. the variant alters gene expression, and the expression of that gene alters the phenotype). Therefore, if a gene within a QTL interval is cis-regulated, we catagorize it as a high priority candidate.

For each QTL, we identified which, if any, genes within the QTL interval also had a cis-eQTL, and in which tissues an eQTL was seen (using transcriptome data from GeneNetwork). Using this same data, we also identified correlations between expression of each of these genes and the phenotype of interest.

\section{'Gene Friends', or coexpression analysis}

Genes with a cis-eQTL in at least one tissue were further considered for coexpression analysis. The top 10,000 correlations were generated in the tissue with the highest correlation between gene expression and the phenotype of interest. Gene-gene correlations with Sample $p(r)<0.05$ were taken into Webgestalt to perform an over-representation analysis [41, 42, 43, 44]. This results in identification of significantly enriched annotations or pathways, in the genes which co-express with our gene of interest. This can often suggest pathways or networks that the gene is involved in, even if the gene itself has not yet been annotated as part of that network.

\section{Gene variant analysis}

Deep, linked-read sequencing of the 152 members of the BXD family has been carried out using Chromium 10X sequencing (https://www.10xgenomics.com/products/linked-reads), resulting in 5,390,695 SNPs and 733,236 indels which are high confidence, and segregate in the population (i.e. have a minor allele frequency greater than 0.2). These 6 million variants are potential causes of QTLs detected in the BXD family. 
To identify potential effects of these variants, we used the Variant Effect Predictor (VEP) website (http://ensembl.org/Tools/VEP and [45]). All variants within our QTL intervals were extracted from the variant vcf file, and uploaded to VEP. Potentially deleterious variants or variants which impact protein function were identified using the 'Consequence', 'IMPACT', 'SIFT' [46,47] and 'BLOSUM62' [48] annotations.

\section{PheWAS}

Phenome-wide association studies (PheWAS) take a genomic region of interest, and find associations between that region and phenotypes measured in GWAS datasets. We used human PheWAS data for all of the candidate genes in our QTLs to detect the genes with relevant human phenotype associations (i.e. behavioral and neurological phenotypes). A relevant association implies confidence in a candidate gene, and suggests cross-species translatability of the finding. We used online PheWAS tools, GWASatlas (https://atlas.ctglab.nl/PheWAS, [49]) and PheWeb (http://pheweb.sph.umich.edu/) for this study.

\section{Results}

\section{Identification of QTLs}

We first sought to identify novel genetic loci linked to the phenotypes from Philip et al., 2010 [39], that were not found in the original study. Comparing QTL mapping using Haley-Knot ( $\mathrm{H}-\mathrm{K}$; as used previously) and GEMMA, there are 426 traits which had a maximum LRS $<17$ with $\mathrm{H}-\mathrm{K}$ (i.e. non-significant), that now have a maximum $-\log (p)>4$. These new QTL are therefore of interest.

To confirm these, we performed linear mixed model (LMM) QTL mapping in R/qt/2, with permutations. This produced 61 traits which are significant compared to the empirical significance threshold generated by permutations [Supplementary table 3].

Two methods were used to identify QTL of interest. First, we had the group of 61 traits that were significant by permutations. The second method we used was to take advantage of independent traits which share QTL at the same location with suggestive $p$-values $(p<0.63)$. Traits were called as independent if they were carried out in separate groups of animals (e.g. males and females) or if the traits were measured at independent timepoints (e.g. at 10 minutes after treatment and 60 minutes after treatment). We identified 25 QTL for 267 traits [Supplementary table 4].

\section{Novel QTL}


For each of the QTL identified above, we determined if they were reported in Philip et al's original study [39], or if related phenotypes have been reported in the MGI database [50].

Several locomotion related QTL map to Chr1:37.671-78.94 Mb, that were not detected in the Philip et al. study. Previously detected relevant phenotypes associated with this region include the loss of righting induced by ethanol 1 QTL [51] and a vertical clinging QTL [52].

We report a novel QTL on Chr3 (51.723-56.473 Mb) for vertical activity, and on Chr 4 (105.245-114.11 Mb) for locomotion in response to cocaine. Previous studies show a QTL for anxiety in this region of Chr 4 [53]. We also report novel QTLs on Chr 5 for handling induced convulsions as an ethanol reponse (4.468-5.172 Mb) and locomotion in response to cocaine (99.801-101.331 Mb). Finally, there was a novel QTL for locomotion in response to cocaine on Chr 11 (46.361-50.383 Mb) [Supplementary table 4]. Philip's study shows the presence of multiple significant QTL on Chr 13 that include locomotor (Injection stress-induced activation), morphine withdrawal measures such as jumps, defecation and urination, response to sensitivity and anxiety related to acute stress.

Table 1: Summary of novel QTL, not found at the significant or suggestive level in the original paper by Philip et al [39]. The position of the QTL, a summary of the phenotypes within that QTL, and relevant phenotypes found in other studies are shown. Details of all identified QTL are in Supplementary table 5 .

\begin{tabular}{|llllll|}
\hline Chr & $\begin{array}{l}\text { QTL } \\
\text { interval (Mb) }\end{array}$ & $\begin{array}{l}\text { confidence } \\
\text { phenotype }\end{array}$ & $\begin{array}{l}\text { Summary } \\
\text { Phenotype }\end{array}$ & $\begin{array}{l}\text { Relevant } \\
\text { phenotype }\end{array}$ \\
\hline Chr1 & $37.671-78.94$ & Locomotion & $\begin{array}{l}\text { Loss of righting induced } \\
\text { by ethanol }\end{array}$ & 8974320 \\
\hline Chr1 & $37.671-78.94$ & Locomotion & Vertical clinging & 10086232 \\
\hline Chr1 & $68.798-80.329$ & Cocaine & and & $\begin{array}{l}\text { Loss of righting induced } \\
\text { by ethanol }\end{array}$ & 16803863 \\
\hline locomotion & Vertical activity & $\begin{array}{l}\text { Loss of righting induced } \\
\text { by ethanol }\end{array}$ & 16803863 \\
\hline Chr3 & $51.723-56.473$ & Vertical activity & & \\
\hline Chr7 & $97.466-104.149$ & & & & \\
\hline Chr12 & $82.859-96.105$ & BXD_11407 & & \\
\hline Chr14 & $109.994-114.751$ & BXD_12023 & & \\
\hline
\end{tabular}




\begin{tabular}{|lllll|}
\hline Chr15 & 71.035-77.148 & $\begin{array}{l}\text { Motor } \\
\text { coordination, } \\
\text { anxiety }\end{array}$ & $\begin{array}{l}\text { Abnormal } \\
\text { fear/anxiety-related } \\
\text { behavior }\end{array}$ & 10556431 \\
\hline Chr16 & $27.038-30.578$ & Morphine & $\begin{array}{l}\text { Abnormal } \\
\text { fear/anxiety-related } \\
\text { behavior }\end{array}$ & 11105392 \\
\hline Chr16 & $27.038-30.578$ & Morphine & $\begin{array}{l}\text { Nicotine induced } \\
\text { locomotor activity }\end{array}$ & 10556431 \\
\hline
\end{tabular}

\section{Candidate causal genes within novel QTL}

We concentrated on a subset of six novel QTL which contained less than 100 genes. These QTL are more amenable to finding plausible candidate genes using bioinformatic methods. We reduce the likelihood of finding false positives, and these large QTL are more likely to be due to two or more variants in different genes both contributing to the phenotype. The advantage of families of isogenic strains, like the BXD, is that more strains could be phenotyped, reducing the size of these QTL regions, and allowing greater precision. We leave these large QTLs to future studies.

The 'small' QTL we use as examples here were: Chr3:51.723-56.473 Mb, Chr 5:4.468-5.172 Mb, Chr5:99.801-101.331 Mb, Chr9:45.671-48.081 Mb, Chr11:62.923-65.082 Mb and Chr 14:109.994-114.751 Mb [Supplementary table 4]

We used several tools to narrow down potential candidate genes within these QTL. Variants can change phenotype in two main ways: they can either change gene expression, or can change protein function.

To look for variants altering gene expression, we first looked for genes within our QTL regions which has a local or cis-eQTL. Cis-eQTL demonstrate that there are variants in or close to a gene that cause changes in that gene's expression. This is useful, since it clearly shows that a variant in the eQTL region has a regulatory effect. Therefore, genes with a cis-eQTL are interesting candidate genes.

The next step is to see if expression of these genes correlates with the phenotype(s) of interest. This would suggest a chain of causality: A variant within a gene causes a change in its expression, and the expression of that gene correlates with expression of a trait of interest. To do this, we created a correlation matrix between all genes within a QTL with a cis-eQTL in any brain tissue [Supplementary table 6] and the phenotypes that contributed to the QTL 
[Supplementary table 6]. Any gene with a cis-eQTL and a significantly correlated expression was considered a good candidate. If the gene only had a cis-eQTL and correlation in a single brain region, then it suggested that this brain region might also be of interest for the phenotype (adding another link to this chain).

The QTL region for vertical activity (Chr 3 51.723-56.473 Mb), has 60 genes among which six genes show presence of cis-eQTLs [Supplementary table 6]. No relevant functional annotations (Gene Ontology) have been reported yet. Dclk1 (location of cis-eQTL: Chr3 $55.52 \mathrm{Mb}$ ) variants were previously reported to be associated across Schizophrenia and Attention Deficit Hyperactivity Disorder [54]. The same gene has been described as a candidate gene for inflammatory nociception [55]. Trpc4 (location of cis-eQTL: Chr $354.266176 \mathrm{Mb}$ ) may be involved in the regulation of anxiety-related behaviors [56].

The QTL region for handling induced convulsions (ethanol response; Chr 5 4.468-5.172 Mb), two genes ( $F z d 1$ and $C d k 14$ ) with cis-eQTLs among the three present in this region. Fzd1 (location of cis-eQTL: Chr $54.753 \mathrm{Mb}$ ) receptor regulates adult hippocampal neurogenesis [57].

The locomotion in response to cocaine QTL (Chr 5 99.801-101.331 Mb) has ten genes with cis-eQTLs. QTL Analysis of Enoph1 (location of cis-eQTL: Chr $5100.062 \mathrm{Mb}$ ) in mice indicates that it plays a role in stress reactivity [58]. Variants of Coq2 (location of cis-eQTL: Chr 5100.654 $\mathrm{Mb}$ ) contribute to neurodegenerative disorders like Parkinson's disease [59]. Relevant annotations for other genes with cis-eQTLs have not been reported yet by other studies.

The mechanical nociception QTL (Chr 9 45.671-48.081 Mb), incudes five genes with cis-eQTLs. Sik3 (location of cis-eQTL: Chr $946.222 \mathrm{Mb}$ ) is involved in regulating NREM sleep behavior in mice [60]. Cadm1 (location of cis-eQTL: Chr $947.550 \mathrm{Mb}$ ) knockout mice show increased anxiety, impaired social and emotional behaviors and disrupted motor coordination [61].

Analysis of the locomotion in response to cocaine QTL (Chr $1162.923-65.082 \mathrm{Mb}$ ) revealed five genes with cis-eQTLs. Arhgap44 (location of cis-eQTL: Chr $1165.005456 \mathrm{Mb}$ ) has phenotype associations related to abnormal motor learning, abnormal response to novel object, increased grooming behavior and hypoactivity [62]. The brain regions with highest correlation have been added to the supplementary information [Supplementary table 6].

The locomotion in the center QTL (Chr 14 109.994-114.751 Mb), has a single gene with a cis-eQTL, Slitrk6 (location of cis-eQTL: Chr 14 109.231826 Mb). Knockout of this gene has been associated to impaired locomotory behavior and altered responses to a novel environment making this gene a strong candidate [63].

\section{Coexpression networks or 'gene-friends'}


Genes that are coexpressed are often parts of the same pathways or networks, contributing to similar phenotypes. These so-called 'gene-friends' $[64,65]$ can provide insight into the function of an unannotated gene and can be implied from the annotated functions of the genes it coexpresses with. As new omics data are being generated for the BXD all the time (now including methylation, proteomic and metabolic datasets), new gene-friends can be found.

For each of the genes within our QTLs that also has a cis-eQTL in at least one dataset on GeneNetwork, we performed a correlation analysis with all other probes or genes within that dataset. We then performed an enrichment analysis using WebGestalt using all the probes or genes that correlated with our gene of interest (i.e. the gene with a cis-eQTL), and investigated if any of the enriched annotations or pathways were relevant to the QTL.

Highly relevant enriched phenotypes were found in 9430012M22Rik (location of cis-eQTL: Chr 3 $55.291 \mathrm{Mb}$ ). This gene is present in QTL associated with vertical activity (BXD_12023). Genes that correlate with expression of $9430012 \mathrm{M} 22 \mathrm{Rik}$ in the neocortex are enriched for involvement in abnormal locomotor behavior (FDR=1.2056e-9) and abnormal voluntary movement (FDR=7.1848e-10). Other results for this gene that may be relevant include abnormal synaptic transmission and abnormal nervous system physiology [Supplementary table 7]. The genes that correlate with expression of BC033915 (location of cis-eQTL: Chr9 45.671-48.081 Mb) in the hippocampus are enriched for abnormal motor capabilities/coordination/movement (FDR=2.3483E-11). Other relevant results include abnormal brain morphology and abnormal nervous system physiology. Similarly, this analysis has also revealed that genes expressing in the network of Slitrk6 (location of cis-eQTL: Chr $14109.231 \mathrm{Mb}$ ) in the striatum are involved in abnormal locomotor behavior (FDR=6.978E-12) and abnormal voluntary movement (2.9352E-11). Also, the phenotype of QTL containing Slitrk6 is locomotion hence making this gene a good candidate.

Other genes with cis-eQTLs had significant enrinchments that include abnormal brain morphology, abnormal body composition and abnormal nervous system physiology [Supplementary table 7].

\section{Gene variant analysis}

The second method that a variant can alter phenotype, is by changing protein structure or function. To examine this, we took advantage of the deep sequencing available for all BXD strains. We have identified over 5 million common SNPS and small INDELs which segregate within the BXD family (i.e. occur in greater than $>20 \%$ of the population). For each of the 6 QTL identified above, we looked for variants that were predicted to alter protein structure or splicing, or predicted to be deleterious by SIFT or BLOSUM, using the variant effect predictor (VEP). 
The Chr3:53.667-54.942Mb QTL for vertical activity contains predicted deleterious variants in 10 genes: two missense variants in Ccdc169; a missense variant in Ccna1; an inframe insertion in Dclk1; two frameshift variants, a stop loss, and nine missense variants in Frem2; a frameshift variant and six missense variants in Mab21/1; a frameshift variant, a missense variant, eight frameshift variants, two inframe deletions, 18 missense variants, and three stop losses in Nbea; four inframe deletions, an inframe insertion, six missense variants, and a start loss in Postn; a frameshift variant, eight missense variants, a stop gain, and a stop loss in Spg20; and a missense variant in Trpc4.

The Chr5:4.468-5.172Mb QTL for handling-induced convulsion in response to ethanol contains two missense variants in Fzd1, and four missense variants in $C d k 14$.

The Chr5:100.164-100.895Mb QTL for cocaine related phenotypes, contains predicted deleterious variants in 8 genes: three frameshift variants and three missense variants in Cops4; a missense variant and a stop-gain in Enoph1; a frameshift variant and five missense variants in Hnrnpd; three missense variants and a splice donor variant in Hnrnpdl; two frameshift variants, an inframe insertion, 13 missense variants, and a splice donor variant in Hpse; three missense variants in LIN54; a frameshift variant, one inframe deletions, and a splice donor variant in Sec31a; and a missense variant and a splice donor variant in Tmem150c.

The Chr9: 45.671-48.081Mb QTL for mechanical nociception contains predicted deleterious variants three genes: A frameshift variant, two missense variants and a stop loss in 4931429L15Rik; two frameshift variants and five missense variants in Cadm1; and an inframe deletion, three missense variants, and a stop loss in Cep164.

The Chr11:62.923-65.082Mb QTL for nociception contains four frameshift variants, an inframe deletion, and thirteen missense variants in Myocd.

The Chr14:109.994-114.751Mb QTL for locomotion contains a stop loss, three frameshift variants, and 9 missense variants in Slitrk6.

\section{PheWAS analysis of the genes within QTLS}

Another method to identify candidate genes, is to leverage data generated in another population, or another species. Phenome-wide association studies (PheWAS) take a gene or variant of interest, and find all reported associations in GWAS datasets. A number of these GWAS tools exist, using either different methods, or different human cohorts (https://atlas.ctglab.nl/PheWAS, http://pheweb.sph.umich.edu/).

Mouse QTL mapping has high power but low precision (i.e. we can detect a QTL, but do not know which of tens or hundreds of genes is causal), whereas human GWAS has low power but 
high precision (tens or hundreds of thousands of individuals are needed, but candidate regions are often smaller). By combining the power of mouse QTL mapping and the precision of human PheWAS, we are able to do more than either individually.

Candidate genes might show up in our analysis here that did not show up in our above analysis for several reasons, the most common being that gene expression was not measured in the relevant cell type or timepoint.

The QTL for vertical activity (Chr $351.723-56.473 \mathrm{Mb}$ ) includes a number of genes with relevant psychiatric, neurological and cognitive PheWAS hits. Mam/3 is associated with alcohol dependence [66] and depression [67]. Cog6 has significant associations with Depressive symptoms [68] and worrier/anxious feelings [49]. Nbea is associated with nervous feelings [49] and alcohol dependence [66].

All the three genes present in the Chr5 4.468-5.172 Mb QTL (handling-induced convulsions, ethanol response) show significant PheWAS hits for psychiatric traits. Fzd1 (location of cis-eQTL: Chr $54.753 \mathrm{Mb}$ ) is significantly associated with major depressive disorder [69].

In the Chr 5, 99.801-101.331 Mb region the genes Hnrnpd and Lin54 show the highest number of relevant pheWAS hits. Lin54 is associated with conditions like loneliness, anxiety, tension, and sleep related phenotypes $[49,70,71]$.

Cadm1 (Location of cis-eQTL: Chr $947.550 \mathrm{Mb}$ ) gene was found significantly associated with Schizophrenia and other psychiatric disorders [69,72].

Among the genes with cis-eQTLs in Chr 11, Elac2 (location of cis-eQTL: Chr11 64.988 Mb) and Arghap44 have most significant phenotype associations with schizophrenia/bipolar disorder $[72,73]$.

The QTL for locomotion in the center (Chr14 109.994-114.751 Mb) shows two genes with PheWAS hits. Slitrk6 is significantly associated with Parkinson's Disease [74] and bipolar disorder [73]. Slitrk5 has significant associations with various psychiatric traits including anxiety [49], nervous feelings [49] and alcohol dependence [66] [Supplementary Table 8].

\section{Discussion}

We have demonstrated that old data in populations of isogenic strains can be reanalyzed, identifying novel genetic associations, containing novel candidate genes.

Of particular interest is Slitrk6 on Chr14. Slitrk6 (SLIT And NTRK Like Family Member 6) is a protein coding gene. Our analysis strongly shows that abnormality in Slitrk6 is implicated in disrupted locomotor behavior. The presence of cis-eQTL implies that a variant in this gene is 
effecting its expression and the gene is under its own regulation. Being part of a network in the striatum, whose genes are significantly involved in abnormal locomotory behavior and abnormal voluntary movement increases the plausibility. This gene is involved in altering both gene expression and protein structure/function. PheWAS analysis shows that this gene is involved in various neuropsychiatric and neurological phenotypes. The Slitrks have been previously mentioned as prominent candidate genes involved in neuropsychiatric disorders [75]. The members of the Slitrk family have been shown to be widely expressed in the central nervous system, with partially overlapping yet differential patterns of expression [76]. It is worth noting that this gene along with the other candidates have not been reported in Philip et al.'s study.

Another prominent finding is Cadm1 (Cell adhesion molecule 1), a member of the immunoglobulin superfamily, present on Chr9. Its shows the presence of a cis-eQTL and is found to be associated with schizophrenia. Cadm1 knockout mice show anxiety-like behavior under certain conditions like the open-field and light-dark transition tests and motor coordination and gait were impaired in rotarod and footprint tests [61]. The role of CADM1 in relation to prefrontal brain activities, inhibition function, and ADHD, indicating a potential "gene-brain-behavior" relationship was shown by a study that evaluated the association of CADM1 genotype with ADHD, executive function, and regional brain functions [77]. Studies show a connection between ADHD and pain tolerance [78]. Adults with ADHD are comparatively more sensitive to pain. In such cases, Dopamine agonists like methylphenidate (MP) may exert antinociceptive properties [79] and normalize pain perception. Adults and children with ADHD exhibit motor regulation problems which are in turn associated with the pain levels [80].

We discovered a novel QTL regulating handling induced convulsions after ethanol treatment (BXD_11635) on Chr5:4.468-5.172. Only three genes are within the confidence interval for this QTL, and two of which, Fzd1 and $C d k 14$, have cis-eQTL and predicted deleterious variants. Interestingly, $C d k 14$ is regulated by the anticonvulsant drug valproic acid $[81,82,83]$, and is up-regulated in malaria patients who experience febrile convulsions [84].

We have shown that by using families of isogenic strains, we can not only go back and discover new phenotype-genotype associations that were not previously found, but that we can find highly plausible candidate genes within these novel QTL.

\section{Supplementary Materials}

Supplementary table 1: Phenotype data and reanalysis with new tools and genotypes

For each of the traits obtained from Philip et al. 2010, H-K mapping results from Philip's study and corresponding GEMMA results of Max $-\log (p)$, Max $-\log (p)$ Peak location and Max LRS value from our study are shown. 
Supplementary table 2: Significant traits with QTL regions.

QTL confidence intervals (QTL start region and QTL end region) for each of significant traits determined by 1.5 LOD or $1.5-\log (p)$ drop and number of genes in each of these regions are shown.

Supplementary table 3: Significant traits (H-K Vs GEMMA and R/qt/2)

A total of 426 significant traits which had a maximum LRS $<17$ with $\mathrm{H}-\mathrm{K}$ (i.e. non-significant), and now have a maximum $-\log (p)>4$ (significant with GEMMA). 61 traits of these are significant with $\mathrm{R} / \mathrm{qt} / 2$ with permutations, as mentioned in the table.

Supplementary table 4: QTLs and respective summaries

QTLs were segregated and identified from significant, independent traits. 25 Novel QTLs detected in this study with the number of genes and phenotypes of these QTLs are shown.

Supplementary table 5: Comparison of QTLs found in this study with Philip et al. and other studies.

The novel QTL found in our study compared to the relevant abnormal behavioral phenotypes found in those QTLs in Philip's study and other studies, if any, are shown.

Supplementary table 6: Genes and cis-eQTLs

Six small QTLs with less than a 100 genes considered for cis-eQTL analysis. Each gene in these QTLs analysed for the presence of cis-eQTL are shown with the location. Correlation matrix generated between the genes in a QTL with cis-eQTL in any brain tissue and the phenotype of that QTL. Brain tissues with highest correlation are also shown.

Supplementary table 7: Coexpression networks or 'gene-friends'

The correlations of genes with cis-eQTLs in the regions of highest correlated brain tissue are analysed in Webgestalt for enriched annotations and pathways. The enrichment results, FDR value, $\mathrm{P}$-value and enrichment ratio are shown for each gene with cis-eQTL.

Supplementary table 8: PheWAS

Results of PheWAS analysis of all the genes present in the small QTLs of our interest are shown containing the psychiatric, neurological and cognitive traits that may be the most relevant to our study. 


\section{Conflict of Interest}

The authors declare no conflicts of interest.

\section{References}

[1] Wilkinson, M. D., Dumontier, M., Aalbersberg, I. J. J., Appleton, G., Axton, M., Baak, A., Blomberg, N., et al. (2016). The FAIR Guiding Principles for scientific data management and stewardship. Scientific data, 3, 160018.

[2] Ashbrook, D. G., Arends, D., Prins, P., Mulligan, M. K., Roy, S., Williams, E. G., Lutz, C. M., et al. (2021). A platform for experimental precision medicine: The extended BXD mouse family. Cell Systems, 12(3), 235-247.e9.

[3] Castle, W. E. (1951). Variation in the hooded pattern of rats, and a new allele of hooded. Genetics, 36(3), 254-266.

[4] Belknap, J. K., Crabbe, J. C., Plomin, R., McClearn, G. E., Sampson, K. E., O’Toole, L. A., \& Gora-Maslak, G. (1992). Single-locus control of saccharin intake in BXD/Ty recombinant inbred (RI) mice: some methodological implications for RI strain analysis. Behavior Genetics, 22(1), 81-100.

[5] Belknap, J. K., Metten, P., Helms, M. L., O’Toole, L. A., Angeli-Gade, S., Crabbe, J. C., \& Phillips, T. J. (1993). Quantitative trait loci (QTL) applications to substances of abuse: physical dependence studies with nitrous oxide and ethanol in BXD mice. Behavior Genetics, 23(2), 213-222.

[6] Dickson, P. E., Miller, M. M., Calton, M. A., Bubier, J. A., Cook, M. N., Goldowitz, D., Chesler, E. J., et al. (2016). Systems genetics of intravenous cocaine self-administration in the BXD recombinant inbred mouse panel. Psychopharmacology, 233(4), 701-714.

[7] Grisel, J. E., Belknap, J. K., O’Toole, L. A., Helms, M. L., Wenger, C. D., \& Crabbe, J. C. (1997). Quantitative trait loci affecting methamphetamine responses in BXD recombinant inbred mouse strains. The Journal of Neuroscience, 17(2), 745-754.

[8] Palmer, A. A., Lessov-Schlaggar, C. N., Ponder, C. A., McKinnon, C. S., \& Phillips, T. J. (2006). Sensitivity to the locomotor-stimulant effects of ethanol and allopregnanolone: a quantitative trait locus study of common genetic influence. Genes, Brain, and Behavior, 5(7), 506-517.

[9] Phillips, T. J., Belknap, J. K., Buck, K. J., \& Cunningham, C. L. (1998). Genes on mouse chromosomes 2 and 9 determine variation in ethanol consumption. Mammalian Genome, 9(12), 936-941. 
[10] Rodriguez, L. A., Plomin, R., Blizard, D. A., Jones, B. C., \& McClearn, G. E. (1994). Alcohol acceptance, preference, and sensitivity in mice. I. Quantitative genetic analysis using BXD recombinant inbred strains. Alcoholism, Clinical and Experimental Research, 18(6), 1416-1422.

[11] Boon, A. C. M., Williams, R. W., Sinasac, D. S., \& Webby, R. J. (2014). A novel genetic locus linked to pro-inflammatory cytokines after virulent H5N1 virus infection in mice. BMC Genomics, 15, 1017.

[12] Grizzle, W. E., Mountz, J. D., Yang, P.-A., Xu, X., Sun, S., Van Zant, G. E., Williams, R. W., et al. (2002). BXD recombinant inbred mice represent a novel T cell-mediated immune response tumor model. International Journal of Cancer, 101(3), 270-279.

[13] Hayes, K. S., Hager, R., \& Grencis, R. K. (2014). Sex-dependent genetic effects on immune responses to a parasitic nematode. BMC Genomics, 15, 193.

[14] Miyairi, I., Ziebarth, J., Laxton, J. D., Wang, X., van Rooijen, N., Williams, R. W., Lu, L., et al. (2012). Host genetics and Chlamydia disease: prediction and validation of disease severity mechanisms. Plos One, 7(3), e33781.

[15] Wang, J., Yoon, T. W., Read, R., Yi, A.-K., Williams, R. W., \& Fitzpatrick, E. A. (2020). Genetic variability of $\mathrm{T}$ cell responses in Hypersensitivity Pneumonitis identified using the BXD genetic reference panel. American Journal of Physiology. Lung Cellular and Molecular Physiology.

[16] Ashbrook, David G, Sharmin, N., \& Hager, R. (2017). Offspring genes indirectly influence sibling and maternal behavioural strategies over resource share. Proceedings. Biological Sciences / the Royal Society, 284(1863).

[17] Ashbrook, David George, Roy, S., Clifford, B. G., Riede, T., Scattoni, M. L., Heck, D. H., Lu, L., et al. (2018). Born to cry: A genetic dissection of infant vocalization. Frontiers in Behavioral Neuroscience, 12, 250.

[18] Dickson, P. E., Roy, T. A., McNaughton, K. A., Wilcox, T. D., Kumar, P., \& Chesler, E. J. (2019). Systems genetics of sensation seeking. Genes, Brain, and Behavior, 18(3), e12519.

[19] Graybeal, C., Bachu, M., Mozhui, K., Saksida, L. M., Bussey, T. J., Sagalyn, E., Williams, R. W., et al. (2014). Strains and stressors: an analysis of touchscreen learning in genetically diverse mouse strains. Plos One, 9(2), e87745.

[20] Knoll, A. T., Jiang, K., \& Levitt, P. (2017). Quantitative trait locus mapping and analysis of heritable variation in affiliative social behavior and co-occurring traits. Genes, Brain, and Behavior, 17(5).

[21] Li, Z., Mulligan, M. K., Wang, X., Miles, M. F., Lu, L., \& Williams, R. W. (2010). A transposon 
in Comt generates mRNA variants and causes widespread expression and behavioral differences among mice. Plos One, 5(8), e12181.

[22] Mulligan, M. K., Abreo, T., Neuner, S. M., Parks, C., Watkins, C. E., Houseal, M. T., Shapaker, T. M., et al. (2019). Identification of a Functional Non-coding Variant in the GABA A Receptor $\alpha 2$ Subunit of the C57BL/6J Mouse Reference Genome: Major Implications for Neuroscience Research. Frontiers in genetics, 10, 188.

[23] Williams, E. G., Mouchiroud, L., Frochaux, M., Pandey, A., Andreux, P. A., Deplancke, B., \& Auwerx, J. (2014). An evolutionarily conserved role for the aryl hydrocarbon receptor in the regulation of movement. PLoS Genetics, 10(9), e1004673.

[24] Houtkooper, R. H., Mouchiroud, L., Ryu, D., Moullan, N., Katsyuba, E., Knott, G., Williams, R. W., et al. (2013). Mitonuclear protein imbalance as a conserved longevity mechanism. Nature, 497(7450), 451-457.

[25] Neuner, S. M., Garfinkel, B. P., Wilmott, L. A., Ignatowska-Jankowska, B. M., Citri, A., Orly, J., Lu, L., et al. (2016). Systems genetics identifies Hp1bp3 as a novel modulator of cognitive aging. Neurobiology of Aging, 46, 58-67.

[26] Roy, S., Sleiman, M. B., Jha, P., Williams, E. G., Ingels, J. F., Chapman, C. J., McCarty, M. S., et al. (2019). Gene-by-environmental modulation of longevity and weight gain in the murine BXD family. BioRxiv.

[27] Sandoval-Sierra, J. V., Helbing, A. H. B., Williams, E. G., Ashbrook, D. G., Roy, S., Williams, R. W., \& Mozhui, K. (2020). Body weight and high-fat diet are associated with epigenetic aging in female members of the BXD murine family. Aging Cell, e13207.

[28] Williams, E. G., Pfister, N., Roy, S., Statzer, C., Ingels, J., Bohl, C., Hasan, M., et al. (2020). The molecular landscape of the aging mouse liver. BioRxiv.

[29] Neuner, S. M., Heuer, S. E., Huentelman, M. J., O’Connell, K. M. S., \& Kaczorowski, C. C. (2019). Harnessing Genetic Complexity to Enhance Translatability of Alzheimer's Disease Mouse Models: A Path toward Precision Medicine. Neuron, 101(3), 399-411.e5.

[30] Neuner, S. M., Heuer, S. E., Zhang, J.-G., Philip, V. M., \& Kaczorowski, C. C. (2019). Identification of Pre-symptomatic Gene Signatures That Predict Resilience to Cognitive Decline in the Genetically Diverse AD-BXD Model. Frontiers in genetics, 10, 35.

[31] Neuner, S. M., Wilmott, L. A., Hoffmann, B. R., Mozhui, K., \& Kaczorowski, C. C. (2017). Hippocampal proteomics defines pathways associated with memory decline and resilience in normal aging and Alzheimer's disease mouse models. Behavioural Brain Research, 322(Pt B), 
288-298.

[32] O'Connell, K. M. S., Ouellette, A. R., Neuner, S. M., Dunn, A. R., \& Kaczorowski, C. C. (2019). Genetic background modifies CNS-mediated sensorimotor decline in the AD-BXD mouse model of genetic diversity in Alzheimer's disease. Genes, Brain, and Behavior, 18(8), e12603.

[33] Rosen, G. D., Pung, C. J., Owens, C. B., Caplow, J., Kim, H., Mozhui, K., Lu, L., et al. (2009). Genetic modulation of striatal volume by loci on Chrs 6 and 17 in BXD recombinant inbred mice. Genes, Brain, and Behavior, 8(3), 296-308.

[34] McKnite, A. M., Perez-Munoz, M. E., Lu, L., Williams, E. G., Brewer, S., Andreux, P. A., Bastiaansen, J. W. M., et al. (2012). Murine gut microbiota is defined by host genetics and modulates variation of metabolic traits. Plos One, 7(6), e3919

[35] Taylor, B. A., Heiniger, H. J., \& Meier, H. (1973). Genetic analysis of resistance to cadmium-induced testicular damage in mice. Proceedings of the Society for Experimental Biology and Medicine. Society for Experimental Biology and Medicine, 143(3), 629-633.

[36] Taylor, B. A., Wnek, C., Kotlus, B. S., Roemer, N., MacTaggart, T., \& Phillips, S. J. (1999). Genotyping new BXD recombinant inbred mouse strains and comparison of BXD and consensus maps. Mammalian Genome, 10(4), 335-348.

[37] Peirce, J. L., Lu, L., Gu, J., Silver, L. M., \& Williams, R. W. (2004). A new set of BXD recombinant inbred lines from advanced intercross populations in mice. BMC Genetics, 5, 7.

[38] Wang, J., Williams, R. W., \& Manly, K. F. (2003). WebQTL: web-based complex trait analysis. Neuroinformatics, 1(4), 299-308.

[39] Philip, V. M., Duvvuru, S., Gomero, B., Ansah, T. A., Blaha, C. D., Cook, M. N., Hamre, K. M., et al. (2010). High-throughput behavioral phenotyping in the expanded panel of BXD recombinant inbred strains. Genes, Brain, and Behavior, 9(2), 129-159.

[40] Broman, K. W., Gatti, D. M., Simecek, P., Furlotte, N. A., Prins, P., Sen, Ś., Yandell, B. S., et al. (2019). R/qtl2: Software for Mapping Quantitative Trait Loci with High-Dimensional Data and Multiparent Populations. Genetics, 211(2), 495-502.

[41] Liao, Y., Wang, J., Jaehnig, E. J., Shi, Z., \& Zhang, B. (2019). WebGestalt 2019: gene set analysis toolkit with revamped UIs and APIs. Nucleic Acids Research, 47(W1), W199-W205.

[42] Wang, J., Duncan, D., Shi, Z., \& Zhang, B. (2013). WEB-based GEne SeT AnaLysis Toolkit (WebGestalt): update 2013. Nucleic Acids Research, 41(Web Server issue), W77-83.

[43] Wang, J., Vasaikar, S., Shi, Z., Greer, M., \& Zhang, B. (2017). WebGestalt 2017: a more 
comprehensive, powerful, flexible and interactive gene set enrichment analysis toolkit. Nucleic Acids Research, 45(W1), W130-W137.

[44] Zhang, B., Kirov, S., \& Snoddy, J. (2005). WebGestalt: an integrated system for exploring gene sets in various biological contexts. Nucleic Acids Research, 33(Web Server issue), W741-8.

[45] McLaren, W., Gil, L., Hunt, S. E., Riat, H. S., Ritchie, G. R. S., Thormann, A., Flicek, P., et al. (2016). The ensembl variant effect predictor. Genome Biology, 17(1), 122.

[46] Sim, N.-L., Kumar, P., Hu, J., Henikoff, S., Schneider, G., \& Ng, P. C. (2012). SIFT web server: predicting effects of amino acid substitutions on proteins. Nucleic Acids Research, 40(Web Server issue), W452-7.

[47] Ng, P. C., \& Henikoff, S. (2003). SIFT: Predicting amino acid changes that affect protein function. Nucleic Acids Research, 31(13), 3812-3814.

[48] Eddy, S. R. (2004). Where did the BLOSUM62 alignment score matrix come from? Nature Biotechnology, 22(8), 1035-1036.

[49] Watanabe, K., Stringer, S., Frei, O., Umićević Mirkov, M., de Leeuw, C., Polderman, T. J. C., van der Sluis, S., et al. (2019). A global overview of pleiotropy and genetic architecture in complex traits. Nature Genetics, 51(9), 1339-1348.

[50] Eppig, J. T. (2017). Mouse genome informatics (MGI) resource: genetic, genomic, and biological knowledgebase for the laboratory mouse. ILAR journal / National Research Council, Institute of Laboratory Animal Resources, 58(1), 17-41.

[51] Bennett, B., Markel, P. D., Beeson, M. A., Gordon, L. G., \& Johnson, T. E. (1994). Mapping quantitative trait loci for ethanol-induced anesthesia in LSxSS recombinant inbred and F2 mice: methodology and results. Alcohol and alcoholism (Oxford, Oxfordshire). Supplement, 2, 79-86.

[52] Le Roy, I., Perez-Diaz, F., Cherfouh, A., \& Roubertoux, P. L. (1999). Preweanling sensorial and motor development in laboratory mice: quantitative trait loci mapping. Developmental Psychobiology, 34(2), 139-158.

[53] Nakamura, K., Xiu, Y., Ohtsuji, M., Sugita, G., Abe, M., Ohtsuji, N., Hamano, Y., et al. (2003). Genetic dissection of anxiety in autoimmune disease. Human Molecular Genetics, 12(10), 1079-1086.

[54] Håvik, B., Degenhardt, F. A., Johansson, S., Fernandes, C. P. D., Hinney, A., Scherag, A., Lybæk, H., et al. (2012). DCLK1 variants are associated across schizophrenia and attention deficit/hyperactivity disorder. Plos One, 7(4), e35424. 
[55] Nair, H. K., Hain, H., Quock, R. M., Philip, V. M., Chesler, E. J., Belknap, J. K., \& Lariviere, W. R. (2011). Genomic loci and candidate genes underlying inflammatory nociception. Pain, 152(3), 599-606.

[56] Riccio, A., Li, Y., Tsvetkov, E., Gapon, S., Yao, G. L., Smith, K. S., Engin, E., et al. (2014). Decreased anxiety-like behavior and $\mathrm{Gaq} / 11$-dependent responses in the amygdala of mice lacking TRPC4 channels. The Journal of Neuroscience, 34(10), 3653-3667.

[57] Mardones, M. D., Andaur, G. A., Varas-Godoy, M., Henriquez, J. F., Salech, F., Behrens, M. I., Couve, A., et al. (2016). Frizzled-1 receptor regulates adult hippocampal neurogenesis. Molecular Brain, 9, 29.

[58] Barth, A., Bilkei-Gorzo, A., Drews, E., Otte, D. M., Diaz-Lacava, A., Varadarajulu, J., Turck, C. W., et al. (2013). Analysis of quantitative trait loci in mice suggests a role of Enoph1 in stress reactivity. Journal of Neurochemistry.

[59] Mikasa, M., Kanai, K., Li, Y., Yoshino, H., Mogushi, K., Hayashida, A., Ikeda, A., et al. (2018). COQ2 variants in Parkinson's disease and multiple system atrophy. Journal of Neural Transmission, 125(6), 937-944.

[60] Funato, H., Miyoshi, C., Fujiyama, T., Kanda, T., Sato, M., Wang, Z., Ma, J., et al. (2016). Forward-genetics analysis of sleep in randomly mutagenized mice. Nature, 539(7629), 378-383.

[61] Takayanagi, Y., Fujita, E., Yu, Z., Yamagata, T., Momoi, M. Y., Momoi, T., \& Onaka, T. (2010). Impairment of social and emotional behaviors in Cadm1-knockout mice. Biochemical and Biophysical Research Communications, 396(3), 703-708.

[62] Sarowar, T., Grabrucker, S., Föhr, K., Mangus, K., Eckert, M., Bockmann, J., Boeckers, T. M., et al. (2016). Enlarged dendritic spines and pronounced neophobia in mice lacking the PSD protein RICH2. Molecular Brain, 9, 28.

[63] Matsumoto, Y., Katayama, K., Okamoto, T., Yamada, K., Takashima, N., Nagao, S., \& Aruga, J. (2011). Impaired auditory-vestibular functions and behavioral abnormalities of Slitrk6-deficient mice. Plos One, 6(1), e16497.

[64] van Dam, S., Cordeiro, R., Craig, T., van Dam, J., Wood, S. H., \& de Magalhães, J. P. (2012). GeneFriends: an online co-expression analysis tool to identify novel gene targets for aging and complex diseases. BMC Genomics, 13, 535.

[65] van Dam, S., Craig, T., \& de Magalhães, J. P. (2015). GeneFriends: a human RNA-seq-based gene and transcript co-expression database. Nucleic Acids Research, 43(Database issue), D1124-32. 
[66] Wang, J. C., Foroud, T., Hinrichs, A. L., Le, N. X. H., Bertelsen, S., Budde, J. P., Harari, O., et al. (2013). A genome-wide association study of alcohol-dependence symptom counts in extended pedigrees identifies C15orf53. Molecular Psychiatry, 18(11), 1218-1224.

[67] Howard, D. M., Adams, M. J., Clarke, T.-K., Hafferty, J. D., Gibson, J., Shirali, M., Coleman, J. R. I., et al. (2019). Genome-wide meta-analysis of depression identifies 102 independent variants and highlights the importance of the prefrontal brain regions. Nature Neuroscience, 22(3), 343-352.

[68] Okbay, A., Baselmans, B. M. L., De Neve, J.-E., Turley, P., Nivard, M. G., Fontana, M. A., Meddens, S. F. W., et al. (2016). Genetic variants associated with subjective well-being, depressive symptoms, and neuroticism identified through genome-wide analyses. Nature Genetics, 48(6), 624-633.

[69] Ripke, S., Wray, N. R., Lewis, C. M., Hamilton, S. P., Weissman, M. M., Breen, G., Byrne, E. M., et al. (2013). A mega-analysis of genome-wide association studies for major depressive disorder. Molecular Psychiatry, 18(4), 497-511.

[70] Day, F. R., Ong, K. K., \& Perry, J. R. B. (2018). Elucidating the genetic basis of social interaction and isolation. Nature Communications, 9(1), 2457.

[71] Jones, S. E., Tyrrell, J., Wood, A. R., Beaumont, R. N., Ruth, K. S., Tuke, M. A., Yaghootkar, H., et al. (2016). Genome-Wide Association Analyses in 128,266 Individuals Identifies New Morningness and Sleep Duration Loci. PLoS Genetics, 12(8), e1006125.

[72] Schizophrenia Working Group of the Psychiatric Genomics Consortium. (2014). Biological insights from 108 schizophrenia-associated genetic loci. Nature, 511(7510), 421-427.

[73] Bipolar Disorder and Schizophrenia Working Group of the Psychiatric Genomics Consortium. Electronic address: douglas.ruderfer@vanderbilt.edu, \& Bipolar Disorder and Schizophrenia Working Group of the Psychiatric Genomics Consortium. (2018). Genomic dissection of bipolar disorder and schizophrenia, including 28 subphenotypes. Cell, 173(7), 1705-1715.e16.

[74] Fung, H.-C., Scholz, S., Matarin, M., Simón-Sánchez, J., Hernandez, D., Britton, A., Gibbs, J. R., et al. (2006). Genome-wide genotyping in Parkinson's disease and neurologically normal controls: first stage analysis and public release of data. Lancet Neurology, 5(11), 911-916.

[75] Proenca, C. C., Gao, K. P., Shmelkov, S. V., Rafii, S., \& Lee, F. S. (2011). Slitrks as emerging candidate genes involved in neuropsychiatric disorders. Trends in Neurosciences, 34(3), 143-153. 
[76] Beaubien, F., \& Cloutier, J.-F. (2009). Differential expression of Slitrk family members in the mouse nervous system. Developmental Dynamics, 238(12), 3285-3296.

[77] Jin, J., Liu, L., Chen, W., Gao, Q., Li, H., Wang, Y., \& Qian, Q. (2019). The Implicated Roles of Cell Adhesion Molecule 1 (CADM1) Gene and Altered Prefrontal Neuronal Activity in Attention-Deficit/Hyperactivity Disorder: A "Gene-Brain-Behavior Relationship"? Frontiers in genetics, 10,882 .

[78] Bou Khalil, R., Khoury, E., \& Richa, S. (2018). The Comorbidity of Fibromyalgia Syndrome and Attention Deficit and Hyperactivity Disorder from a Pathogenic Perspective. Pain Medicine, 19(9), 1705-1709.

[79] Treister, R., Eisenberg, E., Demeter, N., \& Pud, D. (2015). Alterations in pain response are partially reversed by methylphenidate (Ritalin) in adults with attention deficit hyperactivity disorder (ADHD). Pain Practice, 15(1), 4-11.

[80] Stray, L. L., Kristensen, Ø., Lomeland, M., Skorstad, M., Stray, T., \& Tønnessen, F. E. (2013). Motor regulation problems and pain in adults diagnosed with ADHD. Behavioral and Brain Functions, 9, 18.

[81] van Breda, S. G. J., Claessen, S. M. H., van Herwijnen, M., Theunissen, D. H. J., Jennen, D. G. J., de Kok, T. M. C. M., \& Kleinjans, J. C. S. (2018). Integrative omics data analyses of repeated dose toxicity of valproic acid in vitro reveal new mechanisms of steatosis induction. Toxicology, 393, 160-170.

[82] Schulpen, S. H. W., Pennings, J. L. A., \& Piersma, A. H. (2015). Gene expression regulation and pathway analysis after valproic acid and carbamazepine exposure in a human embryonic stem cell based neuro-developmental toxicity assay. Toxicological Sciences, 146(2), 311-320.

[83] Rempel, E., Hoelting, L., Waldmann, T., Balmer, N. V., Schildknecht, S., Grinberg, M., Das Gaspar, J. A., et al. (2015). A transcriptome-based classifier to identify developmental toxicants by stem cell testing: design, validation and optimization for histone deacetylase inhibitors. Archives of Toxicology, 89(9), 1599-1618.

[84] Reuterswärd, P., Bergström, S., Orikiiriza, J., Lindquist, E., Bergström, S., Andersson Svahn, H., Ayoglu, B., et al. (2018). Levels of human proteins in plasma associated with acute paediatric malaria. Malaria Journal, 17(1), 426. 